\title{
Evaluation of general physicians' skills in breaking bad news to the patient based on the SPIKES Questionnaire in Qom, 2016
}

\author{
Hoda Ahmari Tehran ${ }^{1}$, Mandana Shirazi $^{2}$, Ahmad Kachoie $^{3}$, Zohre Khalajinia ${ }^{4}$, Khadijeh Alipour Nodoushan $^{5}$
}

\begin{abstract}
Background: Breaking bad and unpleasant news by physician to patient or his or her family is a key moment in communication between a physician and the patient. It is often necessary for physicians to breaking bad and unpleasant news to the patient or his or her family. The objective of this study was to evaluate the skill of general physicians in breaking bad and unpleasant news to the patient based on the SPIKES questionnaire in educational hospitals of Qom University of Medical Sciences in 2016.

Methods: This descriptive-analytical study was conducted on 200 general physicians. Convenient sampling method was used in this study. Data were collected using standard Spikes Questionnaire and data were analyzed by using descriptive and inferential statistical tests through SPSSv21 software. Results: Most of the subjects were male (69.5\%), married (85.1\%), and had no history of receiving formal education about breaking bad news to the patient. The mean and standard deviation of the subjects were $37.43 \pm 4.02$ years. The mean and standard deviation of the score of the skill of breaking bad news were $63.56 \pm 6.51$. While independent $t$ test showed significant difference in mean and standard deviation of score of the skill of breaking bad news between the two groups $(p<0.05)$, no significant difference was reported between two groups in terms of two variables of gender and clinic place ( $p>0.05$ ). Moreover, using variance analysis, a significant difference was found in mean score of breaking bad news in different age groups with different employment history $(p<0.05)$.

Conclusion: The research results revealed that the skill level of the research samples was relatively at desirable level. Given the lack of receiving forma education by general physicians and the impact of breaking bad news from physician to patients and their caregivers on the type of relationship between the physician and the patient, it is recommended to put more emphasis on continuous education programs, designed especially for general physicians.
\end{abstract}

Keywords: breaking bad news, general physician, SPIKES guideline

\section{INTRODUCTION}

Breaking bad news for patients is one of the major concerns of the physicians. In all medical disciplines, it is sometimes necessary to give bad and unpleasant news to the patient or his or her family (1). Breaking bad news is not so easy task and it is clear that one of the most important skills needed for physicians when they encounter the patient is breaking news on the severity of the diseases (2).

In the past, physicians did not inform the patients on the severe and fatal diseases. They believed that the patient's lack of knowledge of this problem would prevent their despair (1). At current time, informing the patient of all the details of the disease (if patient desires) is a legal and ethical duty for all physicians ( 3 and 4). Based on verse 155 of Bagareh Sura, it is the right of all people to know the facts on themselves and in accordance with divine values, they have right to make decision freely and without compulsion about their future. In addition, Islam strongly emphasizes on the pre-

\footnotetext{
Ph.D. Candidate of Medical Education, Faculty medicine, Tehran University of Medical Sciences, Tehran, Iran.

2 Associate Professor of medical education, Educational Development Centre (EDC), Tehran University of Medical Sciences, Tehran, Iran

3 Assistant Professor of surgery, Qom University of Medical Sciences, Qom, Iran.

4 Assistant Professor of Department of Reproductive Health, Qom University of Medical Sciences, Qom, Iran.

5 Master of Librarianship and Informing, Qom University of Medical Sciences, Qom, Iran.
}

\author{
Correspondence: Ahmad Kachoie \\ Assistant Professor of surgery, Qom University of Medical Sciences, Qom, Iran. \\ E-mail: ahmadkachoie478@gmail.com
}

Received: 23 Jun 2018, Accepted: 30 Nov 2018

(C) 2019 by the authors; licensee Modestum Ltd., UK. This article is an open access article distributed under the terms and conditions of the Creative Commons Attribution License (http://creativecommons.org/licenses/by/4.0/). 
death readiness (4). However, breaking bad news on serious or fatal disease is very annoying for the patient and may lead to inappropriate reactions in addition to causing mental illness in the patient (5). Receiving bad news can be a psychological shock to the patient, leading to concern, worry, and sudden changes in his life $(4,6)$. It is important to give the bad news in a way that patients do not miss their hopes and do not feel depressed (7).

From this perspective, the way of informing the patient of bad news has always been the concern of physicians (8). One of the most important reasons that causes physicians have difficulty in breaking bad news to a patient is the lack of their knowledge on properly reporting of the bad news to a patient and lack of knowledge on its consequence. The physician's skill in breaking the bad news to the patient and his or her caregivers give relief for them and makes the momentary and delayed consequences of the bad news much easier (10). The results of the studies suggest that accepting physicians' behavior by patients is associated with the way of breaking the bad news to patients, that in $95 \%$ of cases, those physicians were accepted by patients who showed emotional and sympathetic behavior with the patients in breaking the bad news (11). It can even be stated that based on the studies conducted, the effective and timely use of communication skills in breaking bad news by physicians will also improve the quality of treatment (12). The way of informing the patient on his or her severe or fatal diseases has never been included in Iran's medical education programs (13). However, major educations are provided to medical students in developed countries (14). The SPIKES model is one of the practical guidelines in breaking the bad news in the world based on a mutual communication model. The steps to provide bad news according to the SPIKES guideline include planning a program for conversation, evaluating patient perceptions, inviting the audience for a conversation, breaking information to the patient, responding emotionally to the patient and planning and summarizing (2). If the physician has adequate knowledge and skills during the sixth phase of the SPIKES guideline, he can success in breaking bad news to the patient and preventing severe mental illnesses (15). While there are other solutions other than the SPIKES method to prepare the patient for bad news (14), their formation are generally due to research which has considered the physicians' views (16-18). Based on the studies, SPIKES is a guideline which can fully meet the needs of patients for receiving the bad news $(2,19)$. Since no study has been conducted to evaluate the skill of general physicians to provide unpleasant and bad news using the SPIKES standard questionnaire in Iran, this research was conducted to evaluate the skill of general physicians in Qom in breaking unpleasant news for patient in the years 2015 and 2016.

\section{METHOD AND MATERIALS}

In this descriptive-analytical and cross-sectional study, data on the performance of 200 general physicians working in public and private health centers of Qom in 2016 on breaking bad news was evaluated using the global SPIKES standard questionnaire using the convenient sampling method. Data were collected by three trained questioners. Individual characteristics included variables such as age, gender, marital status, and employment history and working place. The SPIKES standard questionnaire, which is based on the principles of the Global Calgary-Cambridge Standard Guideline, was introduced by the World Federation of Medical Education in 2004 as a standard clinical guide. Thus, this guideline is considered as standard guideline in breaking interpersonal communication skills in the area of the performance of medical job owners in breaking the bad news. The SPIKES protocol includes two mental and environmental domains with 20 questions. The first 11 questions relate to psychological factors (empathy, knowledge and information) and 9 final questions relate to environmental factors (initial coordination, strategy determination, planning, and professionalism). They are scored in 5-point Likert scale and the maximum score for all questionnaire items is 100. Validity and reliability of this questionnaire in Iran was confirmed by FarokhYar in 2012 (19). After collecting the data, they were entered into SPSS V21 and analyzed by descriptive and inferential statistical tests of one-way ANOVA at a significant level of less than 0.05 .

\section{RESULTS}

In the present study, 200 general physicians completed two-part questionnaire. Majority of the subjects were male (69.5\%), married (85.1\%) and working in private sector (59.2\%). The age of the subjects was between 26 and 65 years with a mean and standard deviation of $39.43 \pm 4.02$.

The mean and standard deviation of the score for breaking the bad news were $63.56 \pm 6.15$ in the subjects (Table 1). The results showed that most of the subjects did not receive formal education on breaking the bad news and $83.3 \%$ provided bad news to their patients and their caretakers according to their personal experience. While independent $t$ test showed significant difference between the two groups in terms of mean and standard deviation of score of the skill 
Table 1: The score obtained by physicians in each domain and in total (mean and standard deviation) and the significance level

\begin{tabular}{clc}
\hline Domain & Sub-domain & Mean and standard deviation of obtained score \\
\hline \multirow{3}{*}{ Environmental } & Initial coordination & $11.85 \pm 1.54$ \\
\cline { 2 - 3 } & Strategy determination & $7.12 \pm 1.56$ \\
\cline { 2 - 3 } & Planning & $6.03 \pm 1.21$ \\
\cline { 2 - 3 } & Professionalism & $4.26 \pm 0.65$ \\
\hline \multirow{2}{*}{} & Sympathy & $6.53 \pm 1.57$ \\
\cline { 2 - 3 } & Knowledge and information & $15.14 \pm 2.43$ \\
\hline & Inviting to information & $7.46 \pm 1.48$ \\
\hline & Total score (0-100) & $63.56 \pm 6.51$ \\
\hline
\end{tabular}

Table 2: Comparison of scores in domains of skills in breaking bad news and total score in terms of gender and marital status

\begin{tabular}{|c|c|c|c|c|c|c|}
\hline \multirow{2}{*}{ Domain } & \multicolumn{2}{|c|}{ Gender } & \multirow{2}{*}{ P-value } & \multicolumn{2}{|c|}{ Marital status } & \multirow{2}{*}{ p-value } \\
\hline & Female & Male & & Single & Married & \\
\hline Initial coordination & $11.45 \pm 1.34$ & $11.37 \pm 1.50$ & 0.14 & $11.37 \pm 1.31$ & $11.67 \pm 1.52$ & 0.032 \\
\hline Strategy determination & $7.02 \pm 1.16$ & $7.11 \pm 1.54$ & 0.074 & $7.21 \pm 1.14$ & $7.41 \pm 1.62$ & 0.034 \\
\hline Planning & $6.03 \pm 1.31$ & $6.17 \pm 1.34$ & 0.102 & $6.23 \pm 1.11$ & $6.56 \pm 1.31$ & 0.004 \\
\hline Professionalism & $4.36 \pm 0.75$ & $4.22 \pm 0.81$ & 0.301 & $4.36 \pm 0.32$ & $4.42 \pm 0.86$ & 0.123 \\
\hline Sympathy & $6.46 \pm 1.43$ & $6.87 \pm 1.56$ & 0.083 & $6.41 \pm 1.41$ & $6.83 \pm 1.62$ & 0.013 \\
\hline Knowledge and information & $15.21 \pm 2.23$ & $15.16 \pm 2.74$ & 0.223 & $15.21 \pm 2.12$ & $15.32 \pm 2.54$ & 0.047 \\
\hline Inviting to information & $7.24 \pm 1.32$ & $7.16 \pm 1.34$ & 0.065 & $7.13 \pm 1.21$ & $7.41 \pm 1.23$ & 0.051 \\
\hline Total score & $63.26 \pm 6.51$ & $63.15 \pm 6.49$ & 0.176 & $62.32 \pm 6.34$ & $63.21 \pm 6.39$ & 0.032 \\
\hline
\end{tabular}

of breaking bad news $(p<0.05)$, no significant difference was reported between two groups of males and females and the clinic place (public or private) ( $p>0.05)$.

In addition, t-test results showed significant difference between the mean score of skill of breaking bad news in two groups under the age of 40 and over 40 years $(p=0.03)$. Moreover, analysis of variance between different groups in terms of work employment variable showed significant difference between the two groups $(p<0.05)$.

Among the 20 SPIKES items, the item "I consider the concerns, worries, and fears of the patient when I report the bad news" with $50 \%$, the item "If the patient's family is available, I console them when breaking the bad news" with $49 \%$, and the item "After informing the cancer disease news for patient, I introduce him a specialist and supportive team" with $49 \%$ had the highest priority of the response by the research subjects.

\section{DISCUSSION}

In the present study, mean and the standard deviation of the skill of breaking bad news by the subjects were 63.56 \pm 6.51 . No study was found in line with the present study, but the research conducted by Rosin et al. (2013) in order to determine and compare the role of health care staff in breaking the bad news to patients on 151 health care professionals ( 51 nurses, 38 physicians and 26 social workers) showed that in comparing the score obtained among three groups studied, physicians obtained higher score in domains of feeling responsibility in breaking the bad news to patients and their families. Moreover, social workers obtained higher scores in breaking psychological support to the patients and their families and nurses obtained higher scores in providing supportive tool $(P=0.000)$.

The results of this research show that breaking good news has caused more mental disorder in social workers than physicians and nurses. All three groups assigned more scores to emotional exhaustion, sadness and acceptance of responsibility this task in the consequence of breaking bad news to patients. Compared to the two groups of physicians and social workers, nurses were more fear of breaking the death to relatives or families of patients and made more effort to escape it. At the end of their research, researchers stated that expansion and development of executive guidelines is helpful in reporting the bad news and breaking death to their patients and their caregivers. Researchers also recommend using simulation and other workshops to train health and medical groups (20). In the present study, there was a significant difference between the two single and married groups in terms of the score of skill of breaking the bad news. In addition, a significant difference was seen among the groups at different ages in terms of mean and standard deviation of scores, but in terms of clinic place, this difference was not statistically significant. In the research conducted by Locatelli et al. (2013), the way of breaking the bad news not only was associated with patient's age and gender, but also the geographical area where they were working (21). 
In this regard, in a descriptive study conducted by Arbabi et al in 2008-2009 on a sample of 100 people (50 physicians and 50 nurses) at Tehran Cancer Institute of Imam Khomeini Hospital, the majority of physicians (86\%) and nurses (74\%) with more age and experience had tendency to report the type of disease diagnosed for patients, while only a few physicians (8\%) were trained in breaking the bad news. In addition, the majority of physicians and nurses preferred to inform patients about the diagnosis of the disease when they are alone or in the presence of their spouses. The researchers concluded that, compared to past, physicians and nurses have more willingness to announce the cancer disease diagnosed for the patients and lack of adequate communication skills in caregivers and their concern about managing emotional reactions of patients reduces their willingness to announce bad news to patients. Thus, providing education for physicians and nurses on the way of breaking the bad news for patients is necessary (22).

The present research revealed a significant in mean score of skill of breaking the bad news among different age groups. Studies have also shown that breaking the bad news is one of the challenging issues of clinical physicians, and many of them, especially young ones, have difficulty in this regard and feel bad when breaking the news (23).

In the present research, majority of the subjects reported that they did not receive formal education on breaking the bad news. Hebert et al conducted a study in 2009 on the need for education to improve the skills and knowledge of oncologists and they realized that majority of the research samples did not receive formal education on improving the skills of breaking the bad news. Majority of the research samples (63\%) stated that it is very important to provide formal educations to improve the oncologists' skills in order to acquire the skills of breaking the bad news, and $34 \%$ also stated that education can be useful and only $3 \%$ of respondents did not believe that education is needed. Moreover, $43 \%$ of them stated that they did not receive any organizational support for education in this regard (24). In proving the results of the Hebert study, the results of the research conducted by Goncaluz et al. (2017) to examine the family physicians' perceptions of bad news in Portugal country showed that $85 \%$ of the samples reported that breaking the bad news is a difficult task. In addition, 78\% felt that they needed for education to announce the bad news for the patients and this education is recommended to provide at the beginning of their education (25). Psychological and behavioral training in the provision of health services, and the evaluation of its results, have been emphasized in various studies (26-29).

\section{CONCLUSION}

The research results suggest that the research samples had relatively good skill level. As majority of the samples stated that they did not receive formal education on the skill of breaking the bad news, it is necessary that medical education planners pay attention to this important issue. Hence, it is recommended that the formal curriculum to be designed for medical students on breaking the bad news to acquire this skill. As limited studies been conducted in this area, it is recommended that similar studies to be carried out in this area in other Iranian universities of medical sciences.

\section{REFERENCES}

1. Fallowfield L, Jenkins V. Communicating sad, bad, and difficult news in medicine. Lancet (London, England). 2004; 363(9405):312-9. https://doi.org/10.1016/S0140-6736(03)15392-5

2. Dean $A$, Willis $S$. The use of protocol in breaking bad news: evidence and ethos. International journal of palliative nursing. 2016 Jun 27;22(6):265-71. https://doi.org/10.12968/ijpn.2016.22.6.265 PMid:27349844

3. Izadi A, Mohammadnejad E, Ehsani S. Nurses' experiences regarding truth telling: a phenomenological study. Iranian Journal of Ethics and history of medicine. 2013;6(1). [Full Text in Persian]

4. Parsa $M$, Bagheri E, Larijani B. Telling bad news to patients and its different aspects. Iranian Journal of Ethics and history of medicine. 2011;4(6):1-14. [Full Text in Persian]

5. Managheb S, Mohammadi M. Knowledge and Attitudes of Clinical Teachers, Family Physicians, and Medical Interns towards Giving Bad News, a Study in Jahrom University of Medical Sciences. Iranian Journal of Teaching in medical science. 2011;11(5):436-43. [Full Text in Persian]

6. Buckman R. Breaking bad news: the S-P-I-K-E-S strategy. Commun Oncol. 2005;2:138-45. https://doi.org/10.1016/S1548-5315(11)70867-1

7. Alshammary SA, Hamdan AB, Tamani JC, Alshuhil A, Ratnapalan S, Alharbi M. Breaking bad news among cancer physicians. Journal of Health Specialties. 2017 Apr 1;5(2):66. https://doi.org/10.4103/jhs.JHS_10_17

8. Barnett MM. Effect of breaking bad news on patients' perceptions of doctors. Journal of the Royal Society of Medicine. 2002 Jul 1;95(7):343-7. https://doi.org/10.1177/014107680209500706 PMid:12091508 PMCid:PMC1279938 
9. Molleman E, Krabbendam PJ, Annyas AA, Koops HS, Sleijfer DT, Vermey A. The significance of the doctor-patient relationship in coping with cancer. Social science \& medicine (1982). 1984;18(6):475-80. https://doi.org/10.1016/0277-9536(84)90003-0

10. Ghafarinejad A, Salari P, Mirzadeh A. How to breaking bad news to patients in Medical Sciences of Kerman University. Medical Journal of Hormozgan. 2006;10(2):179-84. [Full Text in Persian]

11. Munoz Sastre MT, Sorum PC, Mullet E. Breaking bad news: the patient's viewpoint. Health communication. 2011 Oct 1;26(7):649-55. https://doi.org/10.1080/10410236.2011.561919 PMid:21598151

12. Lioyd M, Bor R. Communication skills for medicine. Edinburgh: Churchill Livingston; 2004.

13. Randall TC, Wearn AM. Receiving bad news: patients with haematological cancer reflect upon their experience. Palliative medicine. 2005;19(8):594-601. https://doi.org/10.1191/0269216305pm1080oa PMid:16450876

14. Alexander SC, Keitz SA, Sloane R, Tulsky JA. A controlled trial of a short course to improve residents' communication with patients at the end of life. Academic medicine: Journal of the Association of American Medical Colleges. 2006;81(11):1008-12. https://doi.org/10.1097/01.ACM.0000242580.83851.ad PMid:17065871

15. Arnold R, Lidz C. Clinical aspects of informed consent. New York: MacMillan Library; 1995.

16. Managheb SE, Mosalanejad N. Teaching How to Break Bad News: Comparing Role-Play and Group Discussion on Practice of Medical Interns in Jahrom Medical School. Iranian Journal of Medical Education. 2012;11(7):789797. [Full Text in Persian]

17. Baile WF, Buckman R, Lenzi R, Glober G, Beale EA, Kudelka AP. SPIKES-A six-step protocol for delivering bad news: application to the patient with cancer. The oncologist. 2000;5(4):302-11. https://doi.org/10.1634/theoncologist.5-4-302 PMid:10964998

18. Labaf A, Jahanshir A, Shahvaraninasab A. Difficulties in using Western guidelines for breaking bad news in the emergency department: the necessity of indigenizing guidelines for non-Western countries. Iranian Journal of Ethics and history of medicine. 2014;7(1). [Full Text in Persian]

19. Farokhyar N, Shirazi M, Bahador H, Baradaran H R, Jahanshir A. Assessing the validity and reliability of spikes questionnaires regard in of medical residents awareness breaking bad news in TUMS 2012. Razi J Med Sci 2014;21(122):29-36. [Full Text in Persian]

20. Rassin $M$, Dado KP, Avraham $M$. The role of health care professionals in breaking bad news about death: the perspectives of doctors, nurses and social workers. International journal of caring sciences. 2013 May 1;6(2):22.

21. Locatelli C, Piselli P, Cicerchia M, Repetto L. Physicians' age and sex influence breaking bad news to elderly cancer patients. Beliefs and practices of 50 Italian oncologists: the GIO Ger study. Psycho-Oncology. 2013 May 1;22(5):1112-9. https://doi.org/10.1002/pon.3110 PMid:22678763

22. Arbabi M, Roozdar A, Taher M, Shirzad S, Arjmand M, Mohammadi MR, Nejatisafa AA, Tahmasebi M, Roozdar A. How to break bad news: Physicians' and nurses' attitudes. Iranian journal of psychiatry. 2010;5(4):128. PMid:22952506 PMCid:PMC3395928

23. Spencer J. Learning how to break bad news-More than following the rules. Medical teacher. 2012 May 1;34(5):349-50. https://doi.org/10.3109/0142159X.2012.672778 PMid:22471915

24. Hebert HD, Butera JN, Castillo J, Mega AE. Are we training our fellows adequately in delivering bad news to patients? A survey of hematology/oncology program directors. Journal of palliative medicine. 2009 Dec 1;12(12):1119-24. https://doi.org/10.1089/jpm.2009.0074 PMid:19698025

25. Gonçalves JA, Almeida C, Amorim J, Baltasar R, Batista J, Borrero Y, Fallé JP, Faria I, Henriques M, Maia $H$, Fernandes T. Family physicians' opinions on and difficulties with breaking bad news. Porto Biomedical Journal. 2017 May 22.

26. Tayebi Arasteh M, Pouragha B and Bagheri Kahkesh M. The performance of select universities of medical sciences based on the components affecting medical education [version 1; referees: 2 approved]. F1000Research 2018, 7:301. https://doi.org/10.12688/f1000research.13938.1

27. Ghalesefidi M. J., Maghsoudi J., Pouragha B. Effectiveness of gratitude on psychological well-being and quality of life among hospitalized substance abuse patients. Electronic Journal of General Medicine. 2018. https://doi.org/10.29333/ejgm/94091 
28. Pouragha B, Khabiri R, Pourreza A, Zarei E. Behavior of under the Iranian Social Security Organization-Insured Persons on Utilization of Laboratory and Imaging Services. J Mazandaran Univ Med Sci. 2013;23(106):38-47.

29. Tayebi Arasteh M, Pouragha B and Norouzinia R. Studying the conformity of self-assessment results of higher education lecturers with the assessment by others. International Journal of Pharmaceutical Research. July- Sept 2018;10(3):269-76.

$$
\diamond \diamond \diamond \diamond \diamond \diamond \diamond
$$

http://www.ejgm.co.uk 